\title{
The effect of the geomagnetic field on cosmic ray energy estimates and large scale anisotropy searches on data from the Pierre Auger Observatory
}

The Pierre Auger Collaboration

P. Abreu ${ }^{74}$, M. Aglietta ${ }^{57}$, E.J. Ahn ${ }^{93}$, I.F.M. Albuquerque ${ }^{19}$, D. Allard ${ }^{33}$, I. Allekotte ${ }^{1}$, J. Allen $^{96}$, P. Allison ${ }^{98}$, J. Alvarez Castillo ${ }^{67}$, J. Alvarez-Muñiz ${ }^{84}$, M. Ambrosio ${ }^{50}$, A. Aminaei ${ }^{68}$, L. Anchordoqui ${ }^{109}$, S. Andringa ${ }^{74}$, T. Antičić ${ }^{27}$, A. Anzalone ${ }^{56}$, C. Aramo ${ }^{50}$, E. Arganda ${ }^{81}$, F. Arqueros ${ }^{81}$, H. Asorey ${ }^{1}$, P. Assis ${ }^{74}$, J. Aublin ${ }^{35}$, M. Ave ${ }^{41}$, M. Avenier ${ }^{36}$, G. Avila ${ }^{12}$, T. Bäcker ${ }^{45}$, M. Balzer ${ }^{40}$, K.B. Barber ${ }^{13}$, A.F. Barbosa ${ }^{16}$, R. Bardenet ${ }^{34}$, S.L.C. Barroso ${ }^{22}$, B. Baughman ${ }^{98} f$, J. Bäuml ${ }^{39}$, J.J. Beatty ${ }^{98}$, B.R. Becker ${ }^{106}$, K.H. Becker ${ }^{38}$, A. Bellétoile ${ }^{37}$, J.A. Bellido ${ }^{13}$, S. BenZvi ${ }^{108}$, C. Berat ${ }^{36}$, X. Bertou ${ }^{1}$, P.L. Biermann ${ }^{42}$, P. Billoir ${ }^{35}$, F. Blanco ${ }^{81}$, M. Blanco ${ }^{82}$, C. Bleve ${ }^{38}$, H. Blümer ${ }^{41,} 39$, M. Boháčováa ${ }^{29}$, D. Boncioli ${ }^{51}$, C. Bonifazi ${ }^{25}, 35$, R. Bonino ${ }^{57}$, N. Borodai ${ }^{72}$, J. Brack ${ }^{91}$, P. Brogueira ${ }^{74}$, W.C. Brown ${ }^{92}$, R. Bruijn ${ }^{87}$, P. Buchholz ${ }^{45}$, A. Bueno ${ }^{83}$, R.E. Burton ${ }^{89}$, K.S. Caballero-Mora ${ }^{99}$, L. Caramete ${ }^{42}$, R. Caruso $^{52}$, A. Castellina ${ }^{57}$, O. Catalano ${ }^{56}$, G. Cataldi ${ }^{49}$, L. Cazon ${ }^{74}$, R. Cester ${ }^{53}$, J. Chauvin ${ }^{36}$, S.H. Cheng ${ }^{99}$, A. Chiavassa ${ }^{57}$, J.A. Chinellato ${ }^{20}$, A. Chou $^{93}$, J. Chudoba ${ }^{29}$, R.W. Clay ${ }^{13}$, M.R. Coluccia ${ }^{49}$, R. Conceição ${ }^{74}$, F. Contreras ${ }^{11}$, H. Cook $^{87}$, M.J. Cooper ${ }^{13}$, J. Coppens ${ }^{68}, 70$, A. Cordier ${ }^{34}$, S. Coutu ${ }^{99}$, C.E. Covault ${ }^{89}$, A. Creusot ${ }^{33,79}$, A. Criss ${ }^{99}$, J. Cronin ${ }^{101}$, A. Curutiu ${ }^{42}$, S. Dagoret-Campagne ${ }^{34}$, R. Dallier ${ }^{37}$, S. Dasso ${ }^{8,4}$, K. Daumiller ${ }^{39}$, B.R. Dawson ${ }^{13}$, R.M. de Almeida ${ }^{26}$, M. De Domenico ${ }^{52}$, C. De Donato ${ }^{67,48}$, S.J. de Jong ${ }^{68,70}$, G. De La Vega ${ }^{10}$, W.J.M. de Mello Junior ${ }^{20}$, J.R.T. de Mello Neto ${ }^{25}$, I. De Mitri ${ }^{49}$, V. de Souza ${ }^{18}$, K.D. de Vries ${ }^{69}$, G. Decerprit ${ }^{33}$, L. del Peral ${ }^{82}$, M. del Río ${ }^{51,11}$, O. Deligny ${ }^{32}$, H. Dembinski ${ }^{41}$, N. Dhital ${ }^{95}$, C. Di Giulio ${ }^{47,51}$, J.C. Diaz ${ }^{95}$, M.L. Díaz Castro ${ }^{17}$, P.N. Diep ${ }^{110}$, C. Dobrigkeit ${ }^{20}$, W. Docters ${ }^{69}$, J.C. D’Olivo ${ }^{67}$, P.N. Dong ${ }^{110,32}$, A. Dorofeev ${ }^{91}$, J.C. dos Anjos ${ }^{16}$, M.T. Dova ${ }^{7}$, D. D'Urso ${ }^{50}$, I. Dutan ${ }^{42}$, J. Ebrre ${ }^{29}$, R. Engel ${ }^{39}$, M. Erdmann ${ }^{43}$, C.O. Escobar ${ }^{20}$, J. Espadanal ${ }^{74}$, A. Etchegoyen ${ }^{2}$, P. Facal San Luis ${ }^{101}$, I. Fajardo Tapia $^{67}$, H. Falcke ${ }^{68,71}$, G. Farrar ${ }^{96}$, A.C. Fauth ${ }^{20}$, N. Fazzini ${ }^{93}$, A.P. Ferguson ${ }^{89}$, A. Ferrero ${ }^{2}$, B. Fick ${ }^{95}$, A. Filevich ${ }^{2}$, A. Filipčič ${ }^{78,79}$, S. Fliescher ${ }^{43}$, C.E. Fracchiolla ${ }^{91}$, E.D. Fraenkel ${ }^{69}$, U. Fröhlich ${ }^{45}$, B. Fuchs ${ }^{16}$, R. Gaior ${ }^{35}$, R.F. Gamarra ${ }^{2}$, S. Gambetta ${ }^{46}$, B. García ${ }^{10}$, D. García Gámez ${ }^{34,83}$, D. Garcia-Pinto ${ }^{81}$, A. Gascon ${ }^{83}$, H. Gemmeke ${ }^{40}$, K. Gesterling ${ }^{106}$, P.L. Ghia ${ }^{35,57}$, U. Giaccari ${ }^{49}$, M. Giller ${ }^{73}$, H. Glass ${ }^{93}$, M.S. Gold ${ }^{106}$, G. Golup ${ }^{1}$, F. Gomez Albarracin ${ }^{7}$, M. Gómez Berisso ${ }^{1}$, P. Gonçalves ${ }^{74}$, D. Gonzalez ${ }^{41}$, J.G. Gonzalez ${ }^{41}$, B. Gookin ${ }^{91}$, D. Góra ${ }^{41,72}$, A. Gorgi ${ }^{57}$, P. Gouffon ${ }^{19}$, S.R. Gozzini ${ }^{87}$, E. Grashorn ${ }^{98}$, S. Grebe ${ }^{68,70}$, N. Griffith ${ }^{98}$, M. Grigat ${ }^{43}$, A.F. Grillo ${ }^{58}$, Y. Guardincerri ${ }^{4}$, F. Guarino ${ }^{50}$, G.P. Guedes ${ }^{21}$, A. Guzman ${ }^{67}$, J.D. Hague ${ }^{106}$, P. Hansen ${ }^{7}$, D. Harari ${ }^{1}$, S. Harmsma ${ }^{69}, 70$, T.A. Harrison ${ }^{13}$, J.L. Harton ${ }^{91}$, A. Haungs ${ }^{39}$, T. Hebbeker ${ }^{43}$, D. Heck ${ }^{39}$, A.E. Herve ${ }^{13}$, C. Hojvat ${ }^{93}$, N. Hollon ${ }^{101}$, V.C. Holmes ${ }^{13}$, P. Homola ${ }^{72}$, J.R. Hörandel ${ }^{68}$, A. Horneffer ${ }^{68}$, P. Horvath ${ }^{30}$, M. Hrabovský ${ }^{30,29}$, T. Huege ${ }^{39}$, A. Insolia ${ }^{52}$, F. Ionita ${ }^{101}$, A. Italiano ${ }^{52}$, C. Jarne ${ }^{7}$, S. Jiraskova ${ }^{68}$, M. Josebachuili ${ }^{2}$, K. Kadija ${ }^{27}$, K.H. Kampert ${ }^{38}$, P. Karhan ${ }^{28}$, P. Kasper ${ }^{93}$, B. Kégl ${ }^{34}$, B. Keilhauer ${ }^{39}$, A. Keivani ${ }^{94}$, J.L. Kelley ${ }^{68}$, E. Kemp ${ }^{20}$, R.M. Kieckhafer ${ }^{95}$, H.O. Klages ${ }^{39}$, M. Kleifges ${ }^{40}$, J. Kleinfeller ${ }^{39}$, J. Knapp ${ }^{87}$, D.-H. Koang ${ }^{36}$, K. Kotera ${ }^{101}$, N. Krohm ${ }^{38}$, O. Krömer ${ }^{40}$, D. Kruppke-Hansen ${ }^{38}$, F. Kuehn ${ }^{93}$, D. Kuempel ${ }^{38}$, J.K. Kulbartz ${ }^{44}$, N. Kunka ${ }^{40}$, G. La Rosa ${ }^{56}$, C. Lachaud ${ }^{33}$, P. Lautridou ${ }^{37}$, M.S.A.B. Leão ${ }^{24}$, D. Lebrun ${ }^{36}$, P. Lebrun ${ }^{93}$, M.A. Leigui de Oliveira $^{24}$, A. Lemiere ${ }^{32}$, A. LetessierSelvon ${ }^{35}$, I. Lhenry-Yvon ${ }^{32}$, K. Link ${ }^{41}$, R. López ${ }^{63}$, A. Lopez Agüera ${ }^{84}$, K. Louedec ${ }^{34}$, J. Lozano 
Bahilo $^{83}$, L. Lu ${ }^{87}$, A. Lucero ${ }^{2,57}$, M. Ludwig ${ }^{41}$, H. Lyberis ${ }^{32}$, M.C. Maccarone ${ }^{56}$, C. Macolino ${ }^{35}$, S. Maldera ${ }^{57}$, D. Mandat ${ }^{29}$, P. Mantsch ${ }^{93}$, A.G. Mariazzi ${ }^{7}$, J. Marin ${ }^{11,57}$, V. Marin ${ }^{37}$, I.C. Maris ${ }^{35}$, H.R. Marquez Falcon ${ }^{66}$, G. Marsella ${ }^{54}$, D. Martello ${ }^{49}$, L. Martin ${ }^{37}$, H. Martinez ${ }^{64}$, O. Martínez Bravo $^{63}$, H.J. Mathes ${ }^{39}$, J. Matthews ${ }^{94,100}$, J.A.J. Matthews ${ }^{106}$, G. Matthiae ${ }^{51}$, D. Maurizio ${ }^{53}$, P.O. Mazur ${ }^{93}$, G. Medina-Tanco ${ }^{67}$, M. Melissas ${ }^{41}$, D. Melo ${ }^{2,53}$, E. Menichetti ${ }^{53}$, A. Menshikov ${ }^{40}$, P. Mertsch $^{85}$, C. Meurer ${ }^{43}$, S. Mićanović ${ }^{27}$, M.I. Micheletti ${ }^{9}$, W. Miller ${ }^{106}$, L. Miramonti ${ }^{48}$, L. MolinaBueno $^{83}$, S. Mollerach ${ }^{1}$, M. Monasor ${ }^{101}$, D. Monnier Ragaigne ${ }^{34}$, F. Montanet ${ }^{36}$, B. Morales ${ }^{67}$, C. Morello ${ }^{57}$, E. Moreno ${ }^{63}$, J.C. Moreno ${ }^{7}$, C. Morris $^{98}$, M. Mostafá ${ }^{91}$, C.A. Moura ${ }^{24}, 50$, S. Mueller $^{39}$, M.A. Muller ${ }^{20}$, G. Müller ${ }^{43}$, M. Münchmeyer ${ }^{35}$, R. Mussa ${ }^{53}$, G. Navarra ${ }^{57 \dagger}$, J.L. Navarro ${ }^{83}$,

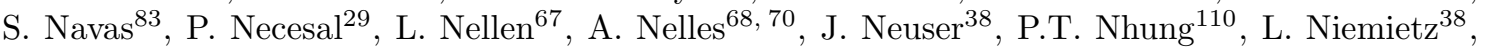
N. Nierstenhoefer ${ }^{38}$, D. Nitz ${ }^{95}$, D. Nosek ${ }^{28}$, L. Nožka $^{29}$, M. Nyklicek ${ }^{29}$, J. Oehlschläger ${ }^{39}$, A. Olinto $^{101}$, P. Oliva ${ }^{38}$, V.M. Olmos-Gilbaja ${ }^{84}$, M. Ortiz ${ }^{81}$, N. Pacheco ${ }^{82}$, D. Pakk Selmi-Dei ${ }^{20}$, M. Palatka ${ }^{29}$, J. Pallotta ${ }^{3}$, N. Palmieri ${ }^{41}$, G. Parente ${ }^{84}$, E. Parizot ${ }^{33}$, A. Parra ${ }^{84}$, R.D. Parsons ${ }^{87}$, S. Pastor ${ }^{80}$,

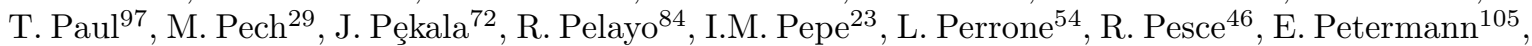
S. Petrera ${ }^{47}$, P. Petrinca ${ }^{51}$, A. Petrolini ${ }^{46}$, Y. Petrov ${ }^{91}$, J. Petrovic ${ }^{70}$, C. Pfendner ${ }^{108}$, N. Phan ${ }^{106}$, R. Piegaia ${ }^{4}$, T. Pierog ${ }^{39}$, P. Pieroni ${ }^{4}$, M. Pimenta ${ }^{74}$, V. Pirronello ${ }^{52}$, M. Platino ${ }^{2}$, V.H. Ponce ${ }^{1}$, M. Pontz ${ }^{45}$, P. Privitera ${ }^{101}$, M. Prouza ${ }^{29}$, E.J. Quel ${ }^{3}$, S. Querchfeld ${ }^{38}$, J. Rautenberg ${ }^{38}$, O. Ravel $^{37}$, D. Ravignani ${ }^{2}$, B. Revenu ${ }^{37}$, J. Ridky ${ }^{29}$, S. Riggi ${ }^{84,52}$, M. Risse ${ }^{45}$, P. Ristori ${ }^{3}$, H. Rivera ${ }^{48}$, V. Rizi ${ }^{47}$, J. Roberts ${ }^{96}$, C. Robledo ${ }^{63}$, W. Rodrigues de Carvalho ${ }^{84}, 19$, G. Rodriguez ${ }^{84}$, J. Rodriguez Martino ${ }^{11}$, J. Rodriguez Rojo ${ }^{11}$, I. Rodriguez-Cabo ${ }^{84}$, M.D. Rodríguez-Frías ${ }^{82}$, G. Ros ${ }^{82}$, J. Rosado ${ }^{81}$, T. Rossler ${ }^{30}$, M. Roth ${ }^{39}$, B. Rouillé-d'Orfeuil ${ }^{101}$, E. Roulet ${ }^{1}$, A.C. Rovero ${ }^{8}$, C. Rühle ${ }^{40}$, F. Salamida ${ }^{47,}{ }^{39}$, H. Salazar ${ }^{63}$, F. Salesa Greus ${ }^{91}$, G. Salina ${ }^{51}$, F. Sánchez ${ }^{2}$, C.E. Santo ${ }^{74}$, E. Santos ${ }^{74}$, E.M. Santos $^{25}$, F. Sarazin ${ }^{90}$, B. Sarkar ${ }^{38}$, S. Sarkar ${ }^{85}$, R. Sato ${ }^{11}$, N. Scharf ${ }^{43}$, V. Scherini ${ }^{48}$, H. Schieler ${ }^{39}$, P. Schiffer ${ }^{43}$, A. Schmidt ${ }^{40}$, F. Schmidt ${ }^{101}$, O. Scholten ${ }^{69}$, H. Schoorlemmer ${ }^{68,70}$, J. Schovancova ${ }^{29}$, P. Schovánek ${ }^{29}$, F. Schröder ${ }^{39}$, S. Schulte ${ }^{43}$, D. Schuster ${ }^{90}$, S.J. Sciutto ${ }^{7}$, M. Scuderi ${ }^{52}$, A. Segreto ${ }^{56}$, M. Settimo ${ }^{45}$, A. Shadkam ${ }^{94}$, R.C. Shellard ${ }^{16,17}$, I. Sidelnik ${ }^{2}$, G. Sigl ${ }^{44}$, H.H. Silva Lopez $^{67}$, A. Śmiałkowski ${ }^{73}$, R. Śmída ${ }^{39,29}$, G.R. Snow ${ }^{105}$, P. Sommers ${ }^{99}$, J. Sorokin ${ }^{13}$, H. Spinka ${ }^{88,93}$, R. Squartini ${ }^{11}$, S. Stanic ${ }^{79}$, J. Stapleton ${ }^{98}$, J. Stasielak ${ }^{72}$, M. Stephan ${ }^{43}$, E. Strazzeri ${ }^{56}$, A. Stutz ${ }^{36}$, F. Suarez ${ }^{2}$, T. Suomijärvi ${ }^{32}$, A.D. Supanitsky ${ }^{8,67}$, T. Šša ${ }^{27}$, M.S. Sutherland ${ }^{94,98}$, J. Swain ${ }^{97}$, Z. Szadkowski ${ }^{73}$, M. Szuba ${ }^{39}$, A. Tamashiro ${ }^{8}$, A. Tapia ${ }^{2}$, M. Tartare ${ }^{36}$, O. Taşcău ${ }^{38}$, C.G. Tavera

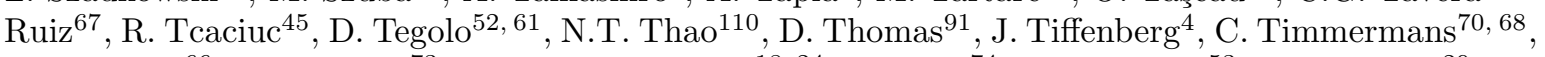
D.K. Tiwari ${ }^{66}$, W. Tkaczyk ${ }^{73}$, C.J. Todero Peixoto ${ }^{18,24}$, B. Tomé ${ }^{74}$, A. Tonachini ${ }^{53}$, P. Travnicek ${ }^{29}$,

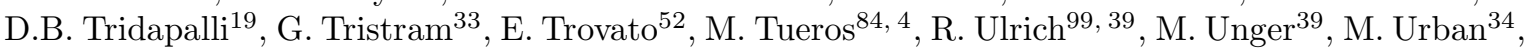
J.F. Valdés Galicia ${ }^{67}$, I. Valiño ${ }^{84,39}$, L. Valore ${ }^{50}$, A.M. van den Berg ${ }^{69}$, E. Varela ${ }^{63}$, B. Vargas

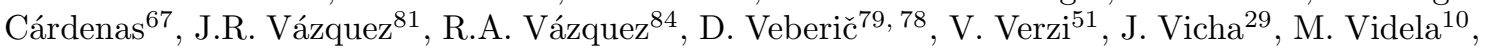
L. Villaseñor ${ }^{66}$, H. Wahlberg ${ }^{7}$, P. Wahrlich ${ }^{13}$, O. Wainberg ${ }^{2}$, D. Walz ${ }^{43}$, D. Warner ${ }^{91}$, A.A. Watson ${ }^{87}$, M. Weber ${ }^{40}$, K. Weidenhaupt ${ }^{43}$, A. Weindl ${ }^{39}$, S. Westerhoff ${ }^{108}$, B.J. Whelan ${ }^{13}$, G. Wieczorek ${ }^{73}$, L. Wiencke ${ }^{90}$, B. Wilczyńska ${ }^{72}$, H. Wilczyński ${ }^{72}$, M. Will ${ }^{39}$, C. Williams ${ }^{101}$, T. Winchen ${ }^{43}$, M.G. Winnick $^{13}$, M. Wommer ${ }^{39}$, B. Wundheiler ${ }^{2}$, T. Yamamoto ${ }^{101}$ a , T. Yapici $^{95}$, P. Younk ${ }^{45}$, G. Yuan ${ }^{94}$, A. Yushkov ${ }^{84,50}$, B. Zamorano ${ }^{83}$, E. Zas ${ }^{84}$, D. Zavrtanik ${ }^{79,78}$, M. Zavrtanik ${ }^{78,79}$, I. Zaw ${ }^{96}$, A. Zepeda ${ }^{64}$, M. Zimbres Silva $^{38,20}$, M. Ziolkowski ${ }^{45}$

1 Centro Atómico Bariloche and Instituto Balseiro (CNEA- UNCuyo-CONICET), San Carlos de Bariloche, Argentina

2 Centro Atómico Constituyentes (Comisión Nacional de Energía Atómica/CONICET/UTN-FRBA), Buenos Aires, Argentina

3 Centro de Investigaciones en Láseres y Aplicaciones, CITEFA and CONICET, Argentina

4 Departamento de Física, FCEyN, Universidad de Buenos Aires y CONICET, Argentina

7 IFLP, Universidad Nacional de La Plata and CONICET, La Plata, Argentina

8 Instituto de Astronomía y Física del Espacio (CONICET- UBA), Buenos Aires, Argentina

${ }^{9}$ Instituto de Física de Rosario (IFIR) - CONICET/U.N.R. and Facultad de Ciencias Bioquímicas y Farmacéuticas U.N.R., Rosario, Argentina 
10 National Technological University, Faculty Mendoza (CONICET/CNEA), Mendoza, Argentina

${ }^{11}$ Observatorio Pierre Auger, Malargüe, Argentina

12 Observatorio Pierre Auger and Comisión Nacional de Energía Atómica, Malargüe, Argentina

${ }^{13}$ University of Adelaide, Adelaide, S.A., Australia

${ }^{16}$ Centro Brasileiro de Pesquisas Fisicas, Rio de Janeiro, RJ, Brazil

${ }^{17}$ Pontifícia Universidade Católica, Rio de Janeiro, RJ, Brazil

${ }^{18}$ Universidade de São Paulo, Instituto de Física, São Carlos, SP, Brazil

${ }^{19}$ Universidade de São Paulo, Instituto de Física, São Paulo, SP, Brazil

${ }^{20}$ Universidade Estadual de Campinas, IFGW, Campinas, SP, Brazil

${ }^{21}$ Universidade Estadual de Feira de Santana, Brazil

${ }^{22}$ Universidade Estadual do Sudoeste da Bahia, Vitoria da Conquista, BA, Brazil

${ }^{23}$ Universidade Federal da Bahia, Salvador, BA, Brazil

${ }^{24}$ Universidade Federal do ABC, Santo André, SP, Brazil

${ }^{25}$ Universidade Federal do Rio de Janeiro, Instituto de Física, Rio de Janeiro, RJ, Brazil

${ }^{26}$ Universidade Federal Fluminense, EEIMVR, Volta Redonda, RJ, Brazil

${ }^{27}$ Rudjer Bošković Institute, 10000 Zagreb, Croatia

28 Charles University, Faculty of Mathematics and Physics, Institute of Particle and Nuclear Physics, Prague, Czech Republic

${ }^{29}$ Institute of Physics of the Academy of Sciences of the Czech Republic, Prague, Czech Republic

${ }^{30}$ Palacky University, RCATM, Olomouc, Czech Republic

32 Institut de Physique Nucléaire d'Orsay (IPNO), Université Paris 11, CNRS-IN2P3, Orsay, France

${ }^{33}$ Laboratoire AstroParticule et Cosmologie (APC), Université Paris 7, CNRS-IN2P3, Paris, France

${ }^{34}$ Laboratoire de l'Accélérateur Linéaire (LAL), Université Paris 11, CNRS-IN2P3, Orsay, France

${ }^{35}$ Laboratoire de Physique Nucléaire et de Hautes Energies (LPNHE), Universités Paris 6 et Paris 7, CNRS-IN2P3, Paris, France

${ }^{36}$ Laboratoire de Physique Subatomique et de Cosmologie (LPSC), Université Joseph Fourier, INPG, CNRS-IN2P3, Grenoble, France

37 SUBATECH, École des Mines de Nantes, CNRS-IN2P3, Université de Nantes, Nantes, France

${ }^{38}$ Bergische Universität Wuppertal, Wuppertal, Germany

${ }^{39}$ Karlsruhe Institute of Technology - Campus North - Institut für Kernphysik, Karlsruhe, Germany

${ }^{40}$ Karlsruhe Institute of Technology - Campus North - Institut für Prozessdatenverarbeitung und Elektronik, Karlsruhe, Germany

${ }^{41}$ Karlsruhe Institute of Technology - Campus South - Institut für Experimentelle Kernphysik (IEKP), Karlsruhe, Germany

${ }^{42}$ Max-Planck-Institut für Radioastronomie, Bonn, Germany

${ }^{43}$ RWTH Aachen University, III. Physikalisches Institut A, Aachen, Germany

${ }^{44}$ Universität Hamburg, Hamburg, Germany

${ }^{45}$ Universität Siegen, Siegen, Germany

${ }^{46}$ Dipartimento di Fisica dell'Università and INFN, Genova, Italy

47 Università dell'Aquila and INFN, L'Aquila, Italy

${ }^{48}$ Università di Milano and Sezione INFN, Milan, Italy

${ }^{49}$ Dipartimento di Fisica dell'Università del Salento and Sezione INFN, Lecce, Italy

50 Università di Napoli "Federico II" and Sezione INFN, Napoli, Italy

${ }^{51}$ Università di Roma II "Tor Vergata" and Sezione INFN, Roma, Italy

${ }^{52}$ Università di Catania and Sezione INFN, Catania, Italy

${ }^{53}$ Università di Torino and Sezione INFN, Torino, Italy

${ }^{54}$ Dipartimento di Ingegneria dell'Innovazione dell'Università del Salento and Sezione INFN, Lecce, Italy 
${ }^{56}$ Istituto di Astrofisica Spaziale e Fisica Cosmica di Palermo (INAF), Palermo, Italy

${ }^{57}$ Istituto di Fisica dello Spazio Interplanetario (INAF), Università di Torino and Sezione INFN, Torino, Italy

${ }^{58}$ INFN, Laboratori Nazionali del Gran Sasso, Assergi (L'Aquila), Italy

${ }^{61}$ Università di Palermo and Sezione INFN, Catania, Italy

${ }^{63}$ Benemérita Universidad Autónoma de Puebla, Puebla, Mexico

${ }^{64}$ Centro de Investigación y de Estudios Avanzados del IPN (CINVESTAV), México, D.F., Mexico

${ }^{66}$ Universidad Michoacana de San Nicolas de Hidalgo, Morelia, Michoacan, Mexico

67 Universidad Nacional Autonoma de Mexico, Mexico, D.F., Mexico

68 IMAPP, Radboud University Nijmegen, Netherlands

${ }^{69}$ Kernfysisch Versneller Instituut, University of Groningen, Groningen, Netherlands

70 Nikhef, Science Park, Amsterdam, Netherlands

${ }^{71}$ ASTRON, Dwingeloo, Netherlands

${ }^{72}$ Institute of Nuclear Physics PAN, Krakow, Poland

${ }^{73}$ University of Łódź, Lódź, Poland

${ }^{74}$ LIP and Instituto Superior Técnico, Technical University of Lisbon, Portugal

78 J. Stefan Institute, Ljubljana, Slovenia

${ }^{79}$ Laboratory for Astroparticle Physics, University of Nova Gorica, Slovenia

${ }^{80}$ Instituto de Física Corpuscular, CSIC-Universitat de València, Valencia, Spain

${ }^{81}$ Universidad Complutense de Madrid, Madrid, Spain

${ }^{82}$ Universidad de Alcalá, Alcalá de Henares (Madrid), Spain

${ }^{83}$ Universidad de Granada \& C.A.F.P.E., Granada, Spain

${ }^{84}$ Universidad de Santiago de Compostela, Spain

${ }^{85}$ Rudolf Peierls Centre for Theoretical Physics, University of Oxford, Oxford, United Kingdom

87 School of Physics and Astronomy, University of Leeds, United Kingdom

88 Argonne National Laboratory, Argonne, IL, USA

${ }^{89}$ Case Western Reserve University, Cleveland, OH, USA

${ }^{90}$ Colorado School of Mines, Golden, CO, USA

${ }^{91}$ Colorado State University, Fort Collins, CO, USA

${ }^{92}$ Colorado State University, Pueblo, CO, USA

${ }^{93}$ Fermilab, Batavia, IL, USA

${ }^{94}$ Louisiana State University, Baton Rouge, LA, USA

${ }^{95}$ Michigan Technological University, Houghton, MI, USA

${ }^{96}$ New York University, New York, NY, USA

97 Northeastern University, Boston, MA, USA

98 Ohio State University, Columbus, OH, USA

${ }^{99}$ Pennsylvania State University, University Park, PA, USA

100 Southern University, Baton Rouge, LA, USA

${ }^{101}$ University of Chicago, Enrico Fermi Institute, Chicago, IL, USA

105 University of Nebraska, Lincoln, NE, USA

${ }^{106}$ University of New Mexico, Albuquerque, NM, USA

108 University of Wisconsin, Madison, WI, USA

109 University of Wisconsin, Milwaukee, WI, USA

${ }^{110}$ Institute for Nuclear Science and Technology (INST), Hanoi, Vietnam

(†) Deceased

(a) at Konan University, Kobe, Japan

(f) now at University of Maryland

Abstract 
We present a comprehensive study of the influence of the geomagnetic field on the energy estimation of extensive air showers with a zenith angle smaller than $60^{\circ}$, detected at the Pierre Auger Observatory. The geomagnetic field induces an azimuthal modulation of the estimated energy of cosmic rays up to the $\sim 2 \%$ level at large zenith angles. We present a method to account for this modulation of the reconstructed energy. We analyse the effect of the modulation on large scale anisotropy searches in the arrival direction distributions of cosmic rays. At a given energy, the geomagnetic effect is shown to induce a pseudo-dipolar pattern at the percent level in the declination distribution that needs to be accounted for. 


\section{Introduction}

High energy cosmic rays generate extensive air showers in the atmosphere. The trajectories of the charged particles of the showers are curved in the Earth's magnetic field, resulting in a broadening of the spatial distribution of particles in the direction of the Lorentz force. While such effects are known to distort the particle densities in a dramatic way at zenith angles larger than $\sim 60^{\circ}$ [1, 2, 3, 4, they are commonly ignored at smaller zenith angles where the lateral distribution function is well described by empirical models of the NKG-type [5, 6, based on a radial symmetry of the distribution of particles in the plane perpendicular to the shower axis.

In this article, we aim to quantify the small changes of the particle densities at ground induced by the geomagnetic field for showers with zenith angle smaller than $\sim 60^{\circ}$, focusing on the impacts on the energy estimator used at the Pierre Auger Observatory. As long as the magnitude of these effects lies well below the statistical uncertainty of the energy reconstruction, it is reasonable to neglect them in the framework of the energy spectrum reconstruction. As the strength of the geomagnetic field component perpendicular to the arrival direction of the cosmic ray, $B_{\mathrm{T}}$, depends on both the zenith and the azimuthal angles $(\theta, \varphi)$ of any incoming shower, these effects are expected to break the symmetry of the energy estimator in terms of the azimuthal angle $\varphi$. Such an azimuthal dependence translates into azimuthal modulations of the estimated cosmic ray event rate at a given energy. For any observatory located far from the Earth's poles, any genuine large scale pattern which depends on the declination translates also into azimuthal modulations of the cosmic ray event rate. Thus to perform a large scale anisotropy measurement it is critical to account for azimuthal modulations of experimental origin and for those induced by the geomagnetic field, as already pointed out in the analysis of the Yakutsk data [7] and the ARGO-YBJ data [8. Hence, this work constitutes an accompanying paper of a search for large scale anisotropies, both in right ascension and declination of cosmic rays detected at the Pierre Auger Observatory, the results of which will be reported in a forthcoming publication.

To study the influence of the geomagnetic field on the cosmic ray energy estimator, we make use of shower simulations and of the measurements performed with the surface detector array of the Pierre Auger Observatory, located in Malargüe, Argentina $\left(35.2^{\circ} \mathrm{S}, 69.5^{\circ} \mathrm{W}\right)$ at $1400 \mathrm{~m}$ a.s.l. 9. The Pierre Auger Observatory is designed to study cosmic rays (CRs) with energies above $\sim 10^{18} \mathrm{eV}$. The surface detector array consists of 1660 water Cherenkov detectors sensitive to the photons and the charged particles of the showers. It is laid out over an area of $3000 \mathrm{~km}^{2}$ on a triangular grid and is overlooked by four fluorescence detectors. The energy at which the detection efficiency of the surface detector array saturates is $\sim 3 \mathrm{EeV}[10$. For each event, the signals recorded in the stations are fitted to find the signal at $1000 \mathrm{~m}$ from the shower core, $S(1000)$, used as a measure of the shower size. The shower size $S(1000)$ is converted to the value $S_{38}$ that would have been expected had the shower arrived at a zenith angle of $38^{\circ} . S_{38}$ is then converted into energy using a calibration curve based on the fluorescence telescope measurements [11.

The influence of the geomagnetic field on the spatial distribution of particles for showers with zenith angle less than $60^{\circ}$ is presented in Section 2, through a toy model aimed at explaining the directional dependence of the shower size $S(1000)$ induced by the geomagnetic field. The observation of this effect in the data of the Pierre Auger Observatory is reported in Section 3 , In Section 4, we quantify the size of the $S(1000)$ distortions with zenith and azimuthal angles by means of end-to-end shower simulations, and then present the procedure to convert the shower

size corrected for the geomagnetic effects into energy using the Constant Intensity Cut method. In Section 5, the consequences on large scale anisotropies are discussed, while systematic uncertainties associated with the primary mass, the primary energy and the number of muons in showers are presented in Section [6. 


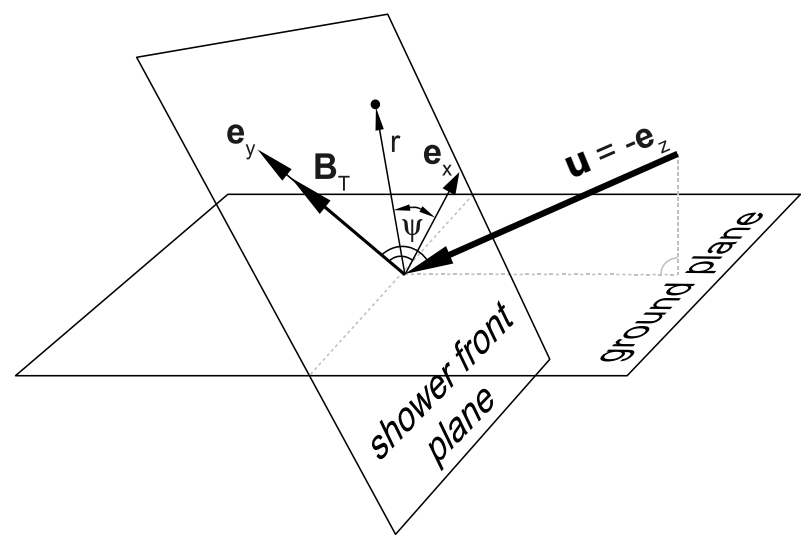

Figure 1: The shower front plane coordinate system [2, 4]: $\mathbf{e}_{z}$ is anti-parallel to the shower direction $\mathbf{u}$, while $\mathbf{e}_{y}$ is parallel to $\mathbf{B}_{\mathrm{T}}$, the projection of the magnetic field $\mathbf{B}$ onto the shower plane $\mathrm{x}-\mathrm{y} . \quad(\psi, r)$ are the polar coordinates in the shower plane.

\section{Influence of the geomagnetic field on extensive air showers}

The interaction of a primary cosmic ray in the atmosphere produces mostly charged and neutral pions, initiating a hadronic cascade. The decay of neutral pions generates the electromagnetic component of the shower, while the decay of the charged pions generates the muonic one. Electrons undergo stronger scattering, so that the electron distribution is only weakly affected by the geomagnetic deflections. Muons are produced with a typical energy $E_{\mu}$ of a few GeV (increasing with the altitude of production). The decay angle between pions and muons is causing only a small additional random deflection, as they almost inherit the transverse momentum $p_{\mathrm{T}}$ of their parents (a few hundred $\mathrm{MeV} / \mathrm{c}$ ) so that the distance of the muons from the shower core scales as the inverse of their energy. While the radial offset of the pions from the shower axis is of the order of a few $10 \mathrm{~m}$, it does not contribute significantly to the lateral distribution of the muons observed on the ground at distances $r \geq 100 \mathrm{~m}$. Hence, at ground level, the angular spread of the muons around the shower axis can be considered as mainly caused by the transverse momentum inherited from the parental pions.

After their production, muons are affected by ionisation and radiative energy losses, decay, multiple scattering and geomagnetic deflections. Below $100 \mathrm{GeV}$, the muon energy loss is mainly due to ionisation and is relatively small (amounting to about $2 \mathrm{MeV} \mathrm{g}^{-1} \mathrm{~cm}^{2}$ ), allowing a large fraction of muons to reach the ground before decaying. Multiple scattering in the electric field of air nuclei randomises the directions of muons to some degree, but the contribution to the total angular divergence of the muons from the shower axis remains small up to zenith angles of the shower-axis of about $80^{\circ}$.

Based on these general considerations, we now introduce a simple toy model aimed at understanding the main features of the muon density distortions induced by the geomagnetic field. We adopt the shower front plane coordinate system depicted in Fig. 1 [2. In the absence of the magnetic field, and neglecting multiple scattering, a relativistic muon of energy $E_{\mu} \simeq c p_{\mu}$ and transverse momentum $p_{\mathrm{T}}$ will reach the shower front plane after traveling a distance $d$ at a position $r$ from the shower axis given by

$$
r \simeq \frac{p_{\mathrm{T}}}{p_{\mu}} d \simeq \frac{c p_{\mathrm{T}}}{E_{\mu}} d
$$

On the other hand, in the presence of the magnetic field, muons suffer additional geomagnetic 


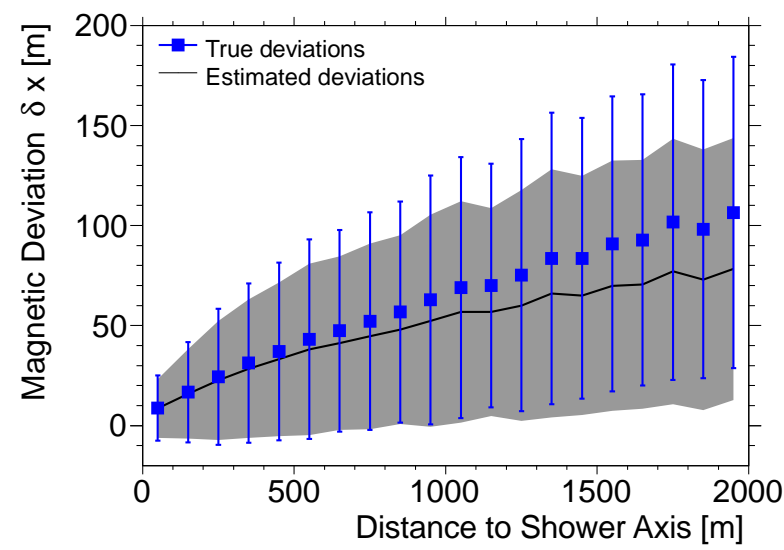

Figure 2: Magnetic deviations as a function of the distance to the shower axis observed on a simulated vertical shower (points). Superimposed are the deviations expected from Eq. (3) (line). The shaded region and the error bars give the corresponding dispersion.

deflections. We treat the geomagnetic field $\mathbf{B}$ in Malargüe as a constant field 1 ,

$$
B=24.6 \mu \mathrm{T}, \quad D_{\mathrm{B}}=2.6^{\circ}, \quad I_{\mathrm{B}}=-35.2^{\circ},
$$

$D_{\mathrm{B}}$ and $I_{\mathrm{B}}$ being the geomagnetic declination and inclination. The deflection of a relativistic muon in the presence of a magnetic field with transverse component $B_{\mathrm{T}}$ can be approximated with

$$
\delta x_{ \pm} \simeq \pm \frac{e c B_{\mathrm{T}} d^{2}}{2 E_{\mu}}
$$

where $e$ is the elementary electric charge and the sign corresponds to positive/negative charged muons. The dependence of the geomagnetic deflections $\delta x \equiv \delta x_{+}=-\delta x_{-}$on the distance to the shower axis $r=\sqrt{x^{2}+y^{2}}$ is illustrated in Fig. 2 obtained by comparing the position of the same muons in the presence or in the absence of the geomagnetic field in a simulated vertical shower of a proton at $5 \mathrm{EeV}$. The deviations expected from the expression for $\delta x_{ \pm}$are also shown in the same graph (solid line). It was obtained by inserting muon energy and distance at the production point of the simulated muons into Eq. (3). It turns out that Eq. (3) estimates rather well the actual deviations, though the distance between the actual and the predicted deviations increases at large $r$. This is mainly because on the one hand $d$ underestimates the actual travel length to a larger extent at larger $r$, while on the other hand the magnetic deviation actually increases while muons gradually lose energy during travel. Hence, from the muon density $\rho_{\mu}(x, y)$ in the transverse plane in the absence of the geomagnetic field, the corresponding density $\bar{\rho}_{\mu}(\bar{x}, \bar{y})$ in the presence of such a field can be obtained by making the following Jacobian transformation, in the same way as in the framework of very inclined showers [2],

$$
\bar{\rho}_{\mu}(\bar{x}, \bar{y})=\left|\frac{\partial(x, y)}{\partial(\bar{x}, \bar{y})}\right| \rho_{\mu}(x(\bar{x}, \bar{y}), y(\bar{x}, \bar{y}))
$$

Here, the term "muon density" refers to the time-integrated muon flux through the transverse shower front plane associated to the air shower, and the barred coordinates represent the positions of the muons in the transverse plane in the presence of the geomagnetic field:

$$
\begin{aligned}
& \bar{x}=x+\delta x_{ \pm}(x, y), \\
& \bar{y}=y .
\end{aligned}
$$

\footnotetext{
${ }^{1}$ In Malargüe the geomagnetic field has varied by about $1^{\circ}$ in direction and $2 \%$ in magnitude over 10 years $[12$.
} 


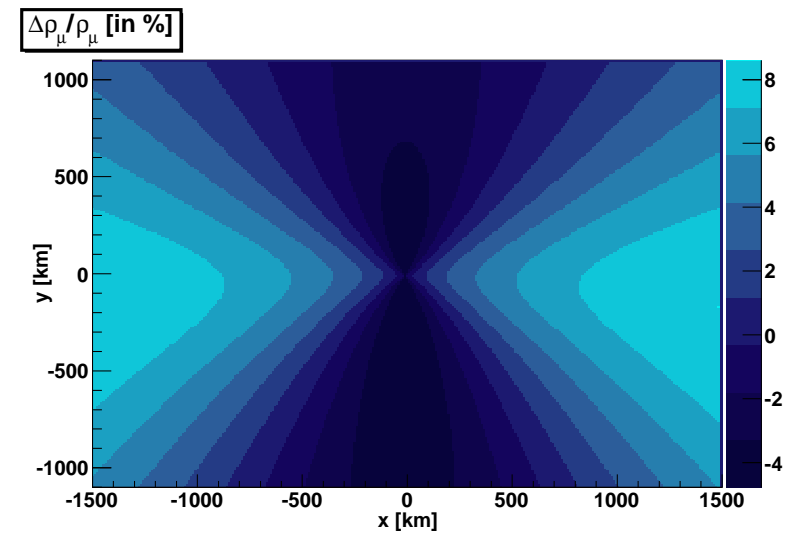

Figure 3: Relative changes of $\Delta \rho_{\mu} / \rho_{\mu}$ in the transverse shower front plane due to the presence of the geomagnetic field, obtained at zenith angle $\theta=60^{\circ}$ and azimuthal angle aligned along $D_{\mathrm{B}}+180^{\circ}$.

Since Eq. (4) induces changes of the shower size $S(1000)$, it is of particular interest to get an approximate relationship between $\rho$ and $\bar{\rho}$ around $1000 \mathrm{~m}$. From Fig. 2, it is apparent that around $1000 \mathrm{~m}$ the mean magnetic deviation is approximately constant over a distance range larger than the size of the deviation. When focusing on the changes of density at $1000 \mathrm{~m}$ from the shower core, it is thus reasonable to neglect the $x$ and $y$ dependence of the deviation $\delta x_{ \pm}$, which allows an approximation of the density $\bar{\rho}_{\mu}(\bar{x}, \bar{y})$ around $1000 \mathrm{~m}$ as

$$
\begin{aligned}
\bar{\rho}_{\mu}(\bar{x}, \bar{y}) & \simeq \rho_{\mu_{+}}\left(\bar{x}-\delta x_{+}, \bar{y}\right)+\rho_{\mu_{-}}\left(\bar{x}-\delta x_{-}, \bar{y}\right) \\
& \simeq \rho_{\mu}(\bar{x}, \bar{y})+\frac{(\delta x)^{2}}{2} \frac{\partial^{2} \rho_{\mu}}{\partial \bar{x}^{2}}(\bar{x}, \bar{y}),
\end{aligned}
$$

where we assumed $\rho_{\mu_{-}}=\rho_{\mu_{+}}=\rho_{\mu} / 2$. The two opposite muon charges cancel out the linear term in $\delta x$ and we see that magnetic effects change the muon density around $1000 \mathrm{~m}$ by a factor proportional to $(\delta x)^{2} \propto B_{T}^{2} \propto \sin ^{2}(\widehat{\mathbf{u}, \mathbf{b}})$, where $\mathbf{u}$ and $\mathbf{b}=\mathbf{B} /|B|$ denote the unit vectors in the shower direction and the magnetic field direction, respectively. This is particularly important with regard to the azimuthal behaviour of the effect, as the azimuthal dependence is contained only in the $B_{\mathrm{T}}^{2}(\theta, \varphi)$ term. This dependency is therefore a generic expectation outlined by this toy model. The model will be verified in Section 4 by making use of complete simulation of showers. On the other hand, the zenith angle dependence relies on other ingredients that we will probe in an accurate way in Section 4, such as the altitude distribution of the muon production and the muon energy distribution.

\section{Observation of geomagnetic effects in the Pierre Auger Observatory data}

To illustrate the differences between $\bar{\rho}_{\mu}$ and $\rho_{\mu}$ described in Eq. (4), the relative changes $\Delta \rho_{\mu} / \rho_{\mu}$ are shown in Fig. 3 in the transverse shower front plane by producing muon maps from simulations at zenith angle $\theta=60^{\circ}$ and azimuthal angle aligned along $D_{\mathrm{B}}+180^{\circ}$ in the presence and in the absence of the geomagnetic field. A predominant quadrupolar asymmetry at the few percent level is visible, corresponding to the separation of positive and negative charges in the direction of the Lorentz force.

This quadrupolar asymmetry is expected to induce to some extent a quadrupolar modulation of the surface detector signals as a function of the polar angle on the ground, defined here as the 


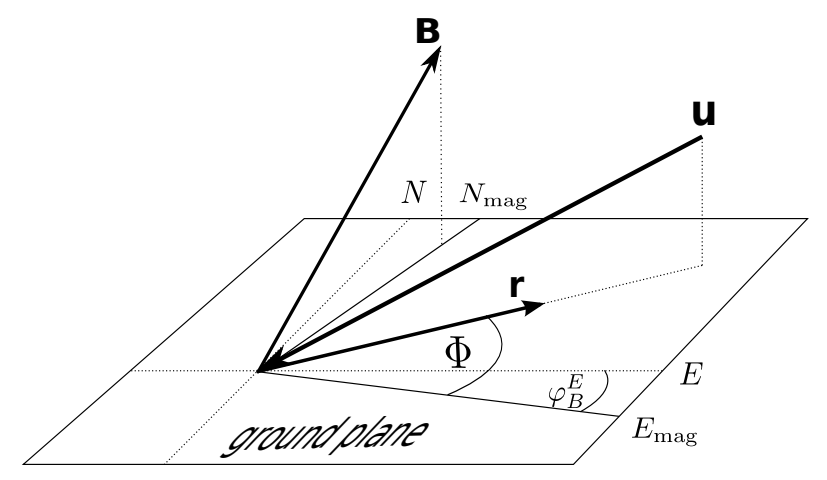

Figure 4: Definition of angle $\Phi$ with respect to the magnetic East $E_{\text {mag }}$ and the shower core for a given shower direction $\mathbf{u}$ and a surface detector at $\mathbf{r}$. The azimuthal angle of the magnetic field vector $\mathbf{B}$ defines the magnetic North $N_{\text {mag }}$.
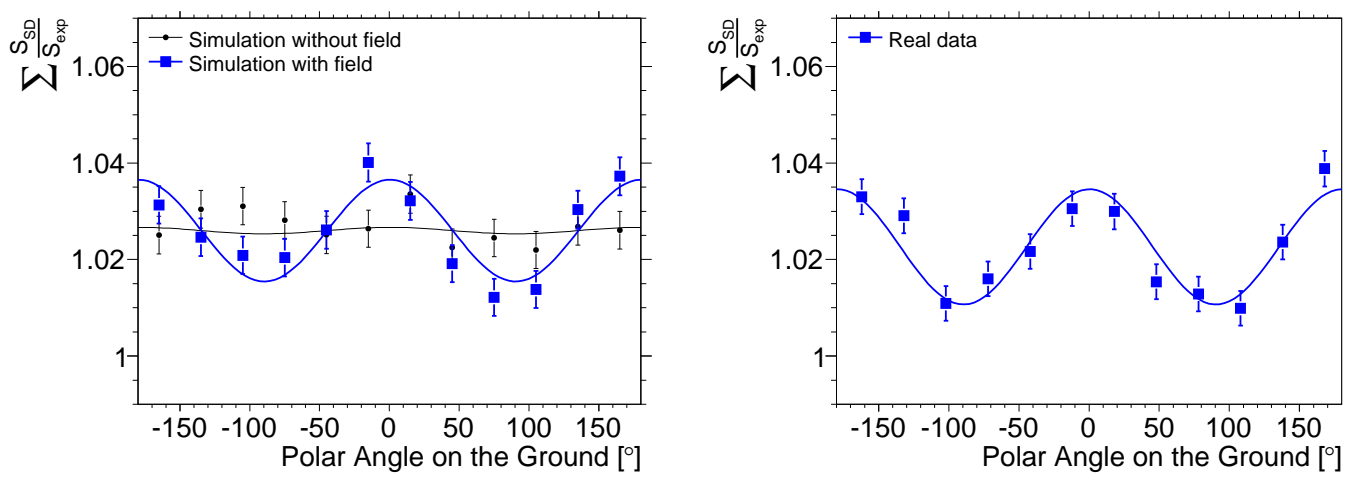

Figure 5: Average ratio of the true signal in each surface detector with respect to the expected one as a function of the polar angle on the ground. Left panel: using simulated showers in the presence (thick points) and in the absence (thin points) of the geomagnetic field. Right panel: using real data above $4 \mathrm{EeV}$. The solid lines give the fit of a quadrupolar modulation to the corresponding points.

angle between the axis given by the shower core and the surface detector, and the magnetic East $\varphi_{\mathrm{B}}^{\mathrm{E}}=-D_{\mathrm{B}}=-2.6^{\circ}$ (Fig. (4). The use of this particular angle, instead of the polar angle $\psi$ which is defined in the shower front plane (see Fig. 1), allows us to remove dipolar asymmetries in the surface detector signals, the origin of which is related to the radial divergence of particles from the shower axis. Such asymmetries cancel out in this analysis, due to the isotropic distribution of the cosmic rays. To demonstrate the geomagnetic effect, we produced a realistic Monte-Carlo simulation using 30000 isotropically distributed showers (with zenith angles less than $60^{\circ}$ ) with random core positions within the array. The injected primary energies were chosen to be greater than $4 \mathrm{EeV}$ (safely excluding angle dependent trigger probability) and distributed according to a power law energy spectrum $d N / d E \propto E^{-\gamma}$ with power index $\gamma=2.7$, so that this shower library is as close as possible to the real data set. To each shower we apply the reconstruction procedure of the surface detector, leading to a fit of the lateral distribution function [11]. The lateral distribution function parametrizes the signal strength in the shower plane, assuming circular shower symmetry. By evaluating the lateral distribution function at the position of the surface detector, we obtain the expected signal $S_{\text {exp }}$. This signal can be compared to the true signal in the surface detector $S_{\mathrm{SD}}$. The ratio between the observed and expected signals as a function of the polar angle on the ground in simulated showers is shown in the left panel of Fig. 5. with (thick points) and without (thin 
points) the geomagnetic field. While a significant quadrupolar modulation with a fixed phase along $D_{\mathrm{B}}$ and amplitude $\simeq(1.1 \pm 0.2) \%$ is observed when the field is on, no such modulation is observed when the field is off $(\simeq(0.1 \pm 0.2) \%)$, as expected. In the right panel, the same analysis is performed on the real data above $4 \mathrm{EeV}$, including again about 30000 showers. A significant modulation of $\simeq(1.2 \pm 0.2) \%$ is observed, agreeing both in amplitude and phase within the uncertainties with the simulations performed in the presence of the geomagnetic field. This provides clear hints of the influence of the geomagnetic field in the Auger data.

Note that this analysis is restricted to surface detectors that are more than $1000 \mathrm{~m}$ away from the shower core. This cut is motivated by Fig. 3 showing that the quadrupolar amplitude is larger at large distances from the shower core. We further require the surface detectors to have signals larger than $4 \mathrm{VEM}^{2}$. This cut is a compromise between keeping good statistics and keeping trigger effects small. Above 4 VEM the measured amplitude does not depend systematically on the signal strength cut. However a cut in the surface detector signals induces a statistical trigger bias because showers with upward signal fluctuations will trigger more readily. This explains the small discrepancy between real and Monte-Carlo data in terms of the global normalisation in Fig. 5 which differs from 1 by $\sim 3 \%$. Cutting at larger signals reduces this discrepancy.

Most importantly, depending on the incoming direction, the quadrupolar asymmetry is also expected to affect the shower size $S(1000)$ and thus the energy estimator as qualitatively described in Eq. (6). Consequently, these effects are expected to modulate the estimated cosmic ray event rate at a given energy as a function of the incoming direction, and in particular to generate a North/South asymmetry in the azimuthal distribution 3 . Such an asymmetry is also expected in the case of a genuine large scale modulation of the flux of cosmic rays. However related analyses of the azimuthal distribution are out of the scope of this paper, and we restrict ourselves in the rest of this article to present a comprehensive study of the geomagnetic distortions of the energy estimator. This will allow us to apply the corresponding corrections in a forthcoming publication aimed at searching for large scale anisotropies.

\section{Geomagnetic distortions of the energy estimator}

\subsection{Geomagnetic distortions of the shower size $S(1000)$}

The toy model presented in Section 2 allows us to understand the main features of the influence of the geomagnetic field on the muonic component of extensive air showers. To get an accurate estimation of the distortions induced by the field on the shower size $S(1000)$ as a function of both the zenith and the azimuthal angles, we present here the results obtained by means of end-toend simulations of proton-initiated showers generated with the AIRES program [14] and with the hadronic interaction model QGSJET [15. We have checked that the results obtained with the CORSIKA program [16] are compatible. We consider a fixed energy $E=5 \mathrm{EeV}$ and seven fixed zenith angles between $\theta=0^{\circ}$ and $\theta=60^{\circ}$. The dependency of the effect in terms of the primary mass and of the number of muons in showers as well as its evolution with energy are sources of systematic uncertainties. The influence of such systematics will be quantified in Section 6 , Within our convention for the azimuthal angle, the azimuthal direction of the magnetic North is $\varphi_{\mathrm{B}}^{\mathrm{N}}=90^{\circ}-D_{\mathrm{B}}=87.4^{\circ}$. The zenith direction of the field is $\theta_{\mathrm{B}}=90^{\circ}-\left|I_{\mathrm{B}}\right|=54.8^{\circ}$.

To verify the predicted behaviour of the shower size shift in terms of $B_{\mathrm{T}}^{2}$, we first show the results of the simulations of 1000 showers at a zenith angle $\theta=\theta_{\mathrm{B}}$ and for two distinct azimuthal angles $\varphi=\varphi_{\mathrm{B}}^{\mathrm{N}}$ and $\varphi=\varphi_{\mathrm{B}}^{\mathrm{N}}+90^{\circ}$. Each shower is then thrown 10 times at the surface detector array with random core positions and reconstructed using exactly the same reconstruction procedure as

\footnotetext{
${ }^{2}$ VEM - Vertical Equivalent Muon - is the average charge corresponding to the Cherenkov light produced by a vertical and central through-going muon in the surface detector. It is the unit used in the evaluation of the signal recorded by the detectors 13 .

${ }^{3}$ The convention we use for the azimuthal angle $\varphi$ is to define it relative to the East direction, counterclockwise.
} 

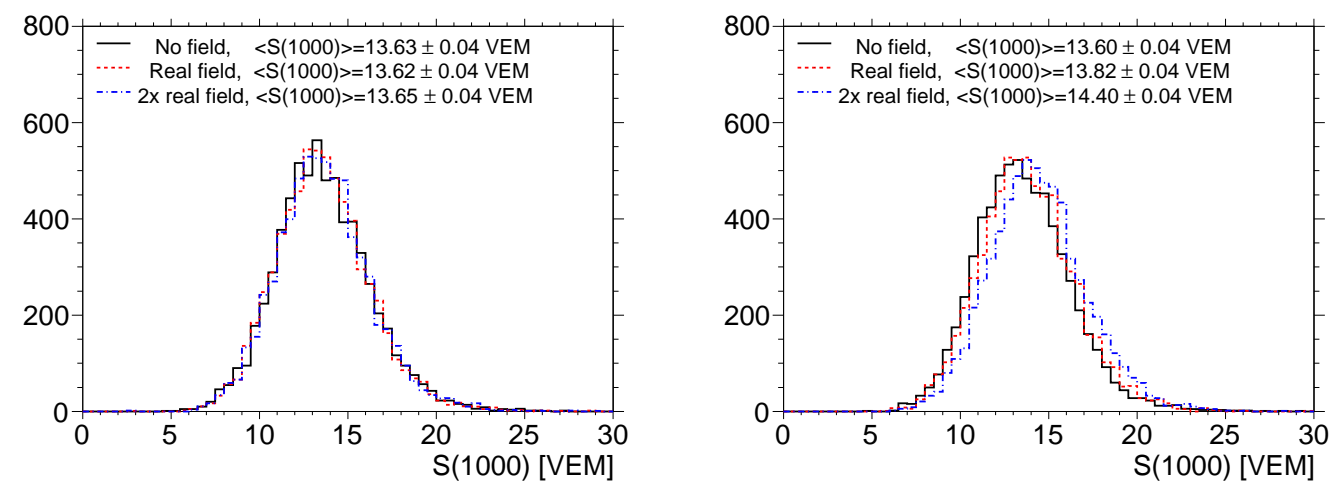

Figure 6: Distributions of shower size $S(1000)$ obtained by simulating showers at zenith angle $\theta=\theta_{\mathrm{B}}$ and azimuthal angle $\varphi_{\mathrm{B}}^{\mathrm{N}}$ (left) and $\varphi_{\mathrm{B}}^{\mathrm{N}}+90^{\circ}$ (right). Thick histogram: no magnetic field. Dotted histogram: real magnetic field in Malargüe. Dashed histogram: twice the real magnetic field in Malargüe.

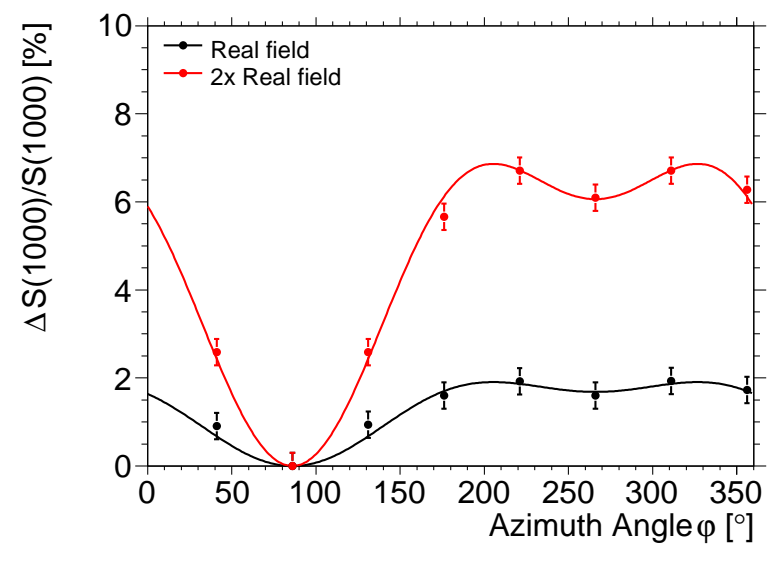

Figure 7: $\Delta S(1000) / S\left(1000\right.$ ) (in \%) as a function of the azimuthal angle $\varphi$, at zenith angle $\theta=\theta_{\mathrm{B}}$ for two different field strengths. Points are obtained by Monte Carlo shower simulation, lines are the expected behavior (see Section 2).

the one applied to real data. For this specific zenith angle $\theta_{\mathrm{B}}$, no shift is expected in the North direction $\varphi_{\mathrm{B}}^{\mathrm{N}}$ as the transverse component of the magnetic field is zero. This is indeed the case as illustrated in the left panel of Fig. 6, showing the distribution of reconstructed $S(1000)$ for three different configurations of the magnetic field: no field, real field in Malargüe, and twice the real field in Malargüe. It can be seen that on average all histograms are - within the statistical uncertainties on the average - centered on the same value. In the right panel of Fig. 6 we repeat the same analysis with the showers generated in the direction $\varphi_{\mathrm{B}}^{\mathrm{N}}+90^{\circ}$. Since the transverse component of the field is now different from zero, a clear relative shift in terms of $\Delta S(1000) / S(1000)$ is observed between the three distributions: the shift is $\simeq 1.6 \%$ between the configurations with and without the field, leading to a discrimination with a significance of $\simeq 5.5 \sigma$, while the shift is $\simeq 6 \%$ between the configurations with twice the real field and without the field leading to a discrimination with a significance of $\simeq 20 \sigma$. It can be noticed that the strength of the shift is thus in overall agreement with the expected scaling $B_{\mathrm{T}}^{2}$.

For the zenith angle $\theta=\theta_{\mathrm{B}}$, in Fig. 7 we show the shift of the mean $S(1000)$ obtained by simulating 1000 showers in the same way as previously for eight different values of the azimuth 


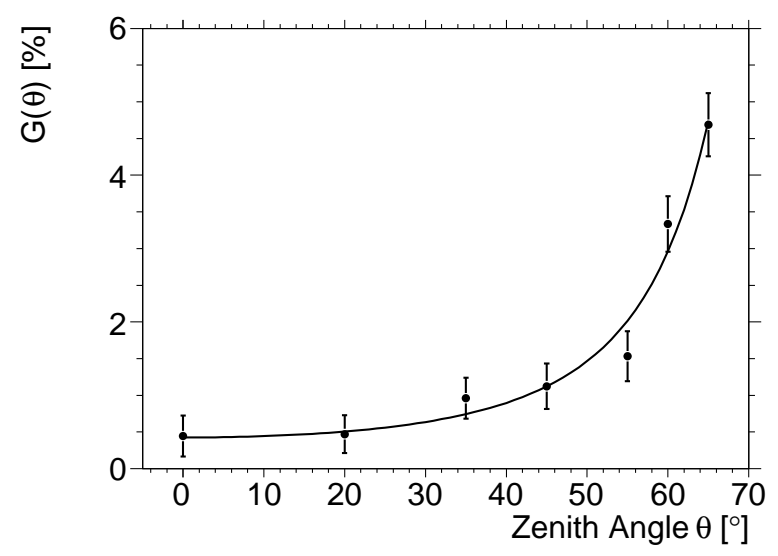

Figure 8: $G(\theta)=\Delta S(1000) / S(1000) / \sin ^{2}(\widehat{\mathbf{u , b}})$ as a function of the zenith angle $\theta$.

angle. Again, the results are displayed for configurations with the real field (bottom) and with twice the real field (top). The expected behaviours in terms of $\Delta S(1000) / S(1000)=G\left(\theta_{\mathrm{B}}\right) \sin ^{2}(\widehat{\mathbf{u}, \mathbf{b}})$ are shown by the continuous curves, where the normalisation factor $G$ is tuned by hand. Clearly, the shape of the curves agrees remarkably well with the Monte Carlo data within the uncertainties. Hence, this study supports the claim that the azimuthal dependence of the shift in $S(1000)$ induced by the magnetic field is proportional to $B_{\mathrm{T}}^{2}(\theta, \varphi)$, in agreement with the expectations provided by general considerations expressed in the previous section on the muonic component of the showers.

The $B_{\mathrm{T}}^{2}$ term encompassing the overall azimuthal dependence at each zenith angle, the remaining shift $G(\theta)=\Delta S(1000) / S(1000) / \sin ^{2}(\widehat{\mathbf{u}, \mathbf{b}})$ depends on the zenith angle through the altitude distribution of the muon production, the muon energy distribution, and the weight of the muonic contribution to the shower size $S(1000)$. Repeating the simulations at different zenith angles, we plot $G$ as a function of the zenith angle in Fig. 8. Due to the increased travel lengths of the muons and due to their larger relative contribution to $S(1000)$ at high zenith angles, the value of $G$ rises rapidly for angles above $\simeq 40^{\circ}$. The superimposed curve is an empirical fit, allowing us to get the following parametrisation of the shower size distortions induced by the geomagnetic field,

$$
\frac{\Delta S(1000)}{S(1000)}(\theta, \varphi)=4.2 \cdot 10^{-3} \cos ^{-2.8} \theta \sin ^{2}(\widehat{\mathbf{u}, \mathbf{b}}) .
$$

\subsection{From shower size to energy}

At the Pierre Auger Observatory, the shower size $S(1000)$ is converted into energy $E$ using a twostep procedure [11. First, the evolution of $S(1000)$ with zenith angle arising from the attenuation of the shower with increasing atmospheric thickness is quantified by applying the Constant Intensity Cut (CIC) method that is based on the (at least approximate) isotropy of incoming cosmic rays. The CIC relates relates $S(1000)$ in vertical and inclined showers through a line of equal intensity in spectra at different zenith angles. This allows us to correct the value of $S(1000)$ for attenuation by computing its value had the shower arrived from a fixed zenith angle, here 38 degrees (corresponding to the median of the angular distribution of events for energies greater than $3 \mathrm{EeV})$. This zenith angle independent estimator $S_{38}$ is defined as $S_{38}=S(1000) / C I C(\theta)$. The calibration of $S_{38}$ with energy $E$ is then achieved using a relation of the form $E=A S_{38}^{B}$, where $A=1.49 \pm 0.06$ (stat) \pm 0.12 (syst) and $B=1.08 \pm 0.01$ (stat) \pm 0.04 (syst) were estimated from the correlation between $S_{38}$ and $E$ in a subset of high quality "hybrid events" measured simultaneously by the surface detector (SD) and the fluorescence detector (FD) [11. In such a sample, $S_{38}$ and E 


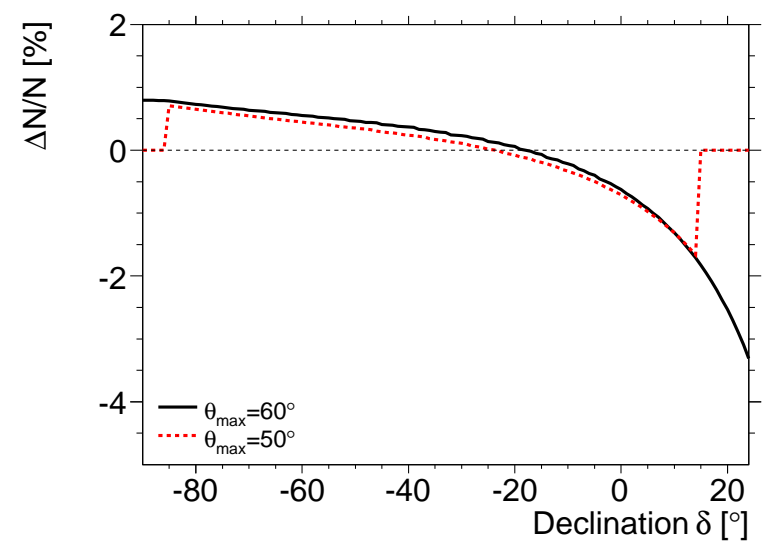

Figure 9: Relative differences $\Delta N / N$ as a function of the declination, for 2 different values of $\theta_{\max }$.

are independently measured, with $S_{38}$ from the SD and $E$ from the FD.

This two-step procedure has an important consequence on the implementation of the energy corrections for the geomagnetic effects. The CIC curve is constructed assuming that the shower size estimator $S(1000)$ does not depend on the azimuthal angle. The induced azimuthal variation of $S(1000)$ due to the geomagnetic effect is thus averaged while the zenith angle dependence of the geomagnetic effects is absorbed when the CIC is implemented. To illustrate this in a simplified way, consider the case in which the magnetic field were directed along the zenith direction (i.e. in the case of a virtual Observatory located at the Southern magnetic pole) so that the transverse component of the magnetic field would not depend on the azimuthal direction of any incoming shower. Then the shift in $S(1000)$ would depend only on the zenith angle in such a way that the Constant Intensity Cut method would by construction absorb the shift induced by $G(\theta)$ into the empirical $C I C(\theta)$ curve, while the empirical relationship $E=A S_{38}^{B}$ would calibrate $S_{38}$ into energy with no need for any additional corrections.

This leads us to implement the energy corrections for geomagnetic effects, relating the energy $E_{0}$ reconstructed ignoring the geomagnetic effects to the corrected energy $E$ by

$$
E=\frac{E_{0}}{(1+\Delta(\theta, \varphi))^{B}}
$$

with

$$
\Delta(\theta, \varphi)=G(\theta)\left[\sin ^{2}(\widehat{\mathbf{u}, \mathbf{b}})-\left\langle\sin ^{2}(\widehat{\mathbf{u}, \mathbf{b}})\right\rangle_{\varphi}\right]
$$

where $\langle\cdot\rangle_{\varphi}$ denotes the average over $\varphi$ and where $B$ is one of the parameters used in the $S_{38}$ to $E$ conversion described above. This expression implies that energies are under-estimated preferentially for showers coming from the northern directions of the array, while they are over-estimated for showers coming from the southern directions, the size of the effect increasing with the zenith angle.

\section{Consequences for large scale anisotropy searches}

\subsection{Impact on the estimated event rate}

To provide an illustration of the impact of the energy corrections for geomagnetic effects, we calculate here, as a function of declination $\delta$, the deviation of the event rate $N_{0}(\delta)$, measured if we 
were not to implement the corrections of the energy estimator by Eq. (8), to the event rate $N(\delta)$ expected from an isotropic background distribution.

The "canonical exposure" [17] holds for a full-time operation of the surface detector array above the energy at which the detection efficiency is saturated over the considered zenith range. In such a case, the directional detection efficiency is simply proportional to $\cos \theta$,

$$
\omega(\theta) \propto \cos (\theta) H\left(\theta-\theta_{\max }\right)
$$

where $H$ is the Heaviside function and $\theta_{\max }$ is the maximal zenith angle considered. The zenith angle is related to the declination $\delta$ and the right ascension $\alpha$ through

$$
\cos \theta=\sin \ell_{\text {site }} \sin \delta+\cos \ell_{\text {site }} \cos \delta \cos \alpha
$$

where $\ell_{\text {site }}$ is the Earth's latitude of the Observatory. The event rate at a given declination $\delta$ and above an energy threshold $E_{\text {th }}$ is obtained by integrating in energy and right ascension $\alpha$,

$$
N(\delta) \propto \int_{E_{\mathrm{th}}}^{\infty} \mathrm{d} E \int_{0}^{2 \pi} \mathrm{d} \alpha \omega(\theta) \frac{\mathrm{d} N(\theta, \varphi, E)}{\mathrm{d} E}
$$

Note that at lower energies this integral acquires an additional energy and angle dependent detection efficiency term $\epsilon(E, \theta, \phi)$. Hereafter we assume that the cosmic ray spectrum is a power law, i.e. $d N / d E \propto E^{-\gamma}$. From Eq. (8) it follows that if the effect of the geomagnetic field were not accounted for, the measured energy spectrum would have a directional modulation given by

$$
\frac{\mathrm{d} N}{\mathrm{~d} E_{0}} \propto[1+\Delta(\theta, \varphi)]^{B(\gamma-1)} E_{0}^{-\gamma} .
$$

This leads to the following measured event rate above a given uncorrected energy $E_{\mathrm{th}}$,

$$
N_{0}(\delta) \propto \int_{E_{\mathrm{th}}}^{\infty} \mathrm{d} E_{0} \int_{0}^{2 \pi} \mathrm{d} \alpha H\left(\cos \theta-\cos \theta_{\max }\right) \cos \theta[1+\Delta(\theta, \varphi)]^{B(\gamma-1)} E_{0}^{-\gamma},
$$

where $\varphi$ is related to $\alpha$ and $\delta$ through

$$
\tan \varphi=\frac{\sin \delta \cos \ell_{\text {site }}-\cos \delta \cos \alpha \sin \ell_{\text {site }}}{\cos \delta \sin \alpha} .
$$

The event rate $N_{0}(\delta)$ as a function of declination is then calculated using Eq. (13) in Eq. (12). The relative difference $\Delta N / N$ is shown in Fig. 9 as a function of the declination, with spectral index $\gamma=2.7$. The energy over-estimation (under-estimation) of events coming preferentially from the Southern (Northern) azimuthal directions, as described in Eq. (8), leads to an effective excess (deficit) of the event rate for $\delta \lesssim-20^{\circ}\left(\delta \gtrsim-20^{\circ}\right)$, with an amplitude of $\simeq 2 \%$ when considering $\theta_{\max }=60^{\circ}$. It is worth noting that this amplitude is reduced to within $1 \%$ when considering $\theta_{\max }=50^{\circ}$, as shown by the dotted line.

\subsection{Impact on dipolar modulation searches}

The pattern displayed in Fig. 9 roughly imitates a dipole with an amplitude at the percent level. To evaluate precisely the impact of this pattern on the assessment of a dipole moment in the reconstructed arrival directions and to probe the statistics needed for the sensitivity to such a spurious pattern, we apply the multipolar reconstruction adapted to the case of a partial sky coverage [18 to mock data sets by limiting the maximum bound of the expansion $L_{\max }$ to 1 (pure dipolar reconstruction). Since the distortions are axisymmetric around the axis defined by the North and South celestial poles, only the multipolar coefficient related to this particular axis is 

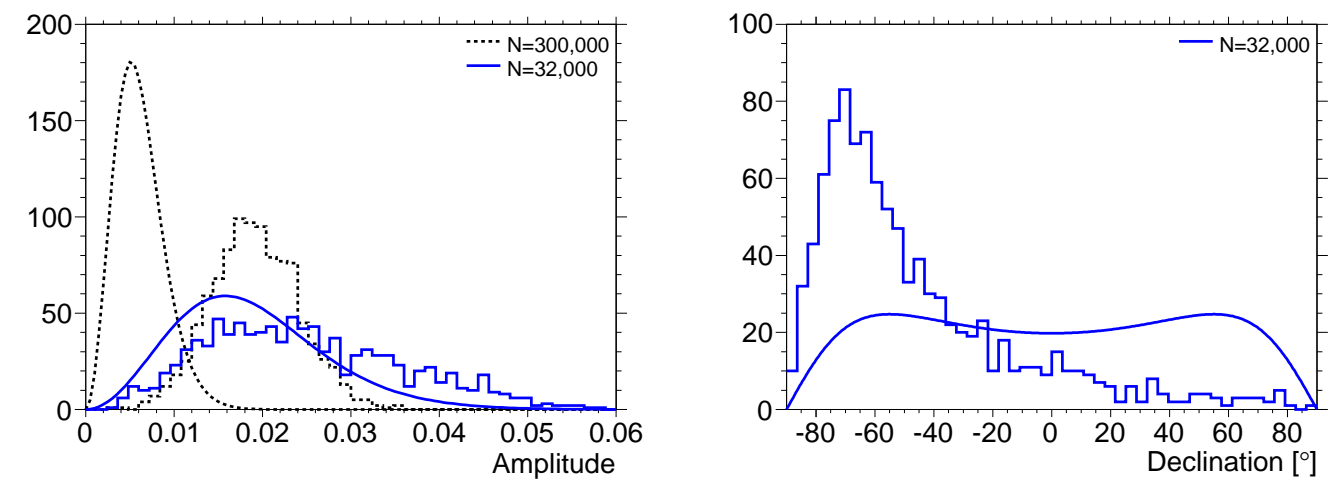

Figure 10: Dipolar reconstruction of arrival directions of mock data sets with event rates distorted by the geomagnetic effects. Left: distributions of amplitudes. Right: distributions of declinations. The smooth lines give the expected distribution in the case of isotropy.

expected to be affected (here: $a_{10}$ ). Consequently, this particular coefficient has impacts on both the amplitude of the reconstructed dipole and its direction with respect to the axis defined by the North and South celestial poles (the technical details of relating the estimation of the multipolar coefficients to the spherical coordinates of a dipole are given in the Appendix).

To simulate the directional distortions induced by Eq. (8), each mock data set is drawn from the event rate $N_{0}(\delta)$ corresponding to the uncorrected energies, and is reconstructed using the canonical exposure in Eq. (10). The results of this procedure applied to 1000 samples are shown in Fig. 10, In the left panel, the distribution of the reconstructed amplitudes $r$ using $N=300000$ events is shown by the dotted histogram. It clearly deviates from the expected isotropic distribution displayed as the dotted curve which corresponds to (see Appendix)

$$
p_{R}(r)=\frac{r}{\sigma \sqrt{\sigma_{z}^{2}-\sigma^{2}}} \operatorname{erfi}\left(\frac{\sqrt{\sigma_{z}^{2}-\sigma^{2}}}{\sigma \sigma_{z}} \frac{r}{\sqrt{2}}\right) \exp \left(-\frac{r^{2}}{2 \sigma^{2}}\right)
$$

where $\operatorname{erfi}(z)=\operatorname{erf}(i z) / i$, and where the width parameters $\sigma$ and $\sigma_{z}$ can be calculated from the exposure function [18. With the particular exposure function used here, it turns out that $\sigma \simeq 1.02 \sqrt{3 / N}$ and $\sigma_{z} \simeq 1.59 \sqrt{3 / N}$. This allows us to estimate the spurious dipolar amplitude4 to be of the order of the mean of the dotted histogram, about $\simeq 1.9 \%$. Consequently, we can estimate that the spurious effect becomes predominant as soon as the mean noise amplitude $\langle r\rangle$ deduced from Eq. (16) is of the order of $1.9 \%$,

$$
\langle r\rangle=\sqrt{\frac{2}{\pi}}\left(\sigma_{z}+\frac{\sigma^{2} \operatorname{arctanh}\left(\sqrt{1-\sigma^{2} / \sigma_{z}^{2}}\right)}{\sqrt{\sigma_{z}^{2}-\sigma^{2}}}\right) \simeq 1.9 \% .
$$

This translates into the condition $N \simeq 32000$ (solid histogram). Using such a number of events, the bias induced on the amplitude reconstruction is illustrated in the same graph by the longer tail of the full histogram with respect to the expected one, and is even more evident in the right panel of Fig. 10, showing the distribution of the reconstructed declination direction of the dipole which already deviates to a large extent from the expected distribution.

\footnotetext{
${ }^{4}$ Due to the partial sky exposure considered here, the estimate of the dipolar amplitude is biased by the higher multipolar orders needed to fully describe $\Delta N / N$ shown in Fig. 10 18. The aim of this calculation is only to provide a quantitative illustration of the spurious measurement which would be performed due to the geomagnetic effects when reconstructing a pure dipolar pattern.
} 


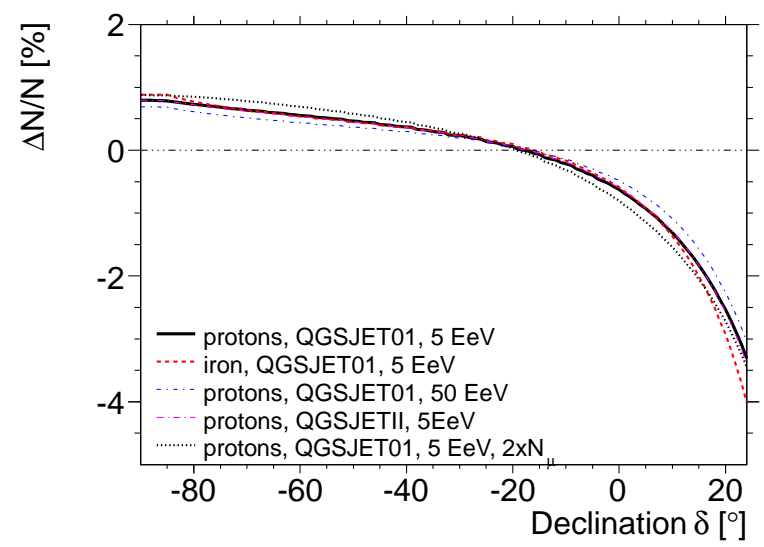

Figure 11: Relative differences $\Delta N / N$ as a function of the declination, for different primary masses, different primary energies, different hadronic models and for increased number of muons in showers.

\section{Systematic uncertainties}

The parametrisation of $G(\theta)$ in Eq. (7) was obtained by means of simulations of proton showers at a fixed energy. The height of the first interaction influences the production altitude of muons detected at $1000 \mathrm{~m}$ from the shower core at the ground level. Moreover, as muons are produced at the end of the hadronic cascade, when the energy of the charged mesons is diminished so much that their decay length becomes smaller than their interaction length (which is inversely proportional to the air density), the energy distribution of muons is also affected by the height of the first interaction. Because the air density is lower in the upper atmosphere, this mechanism results in an increase of the energy of muons. The muonic contribution to $S(1000)$ depends also on both the primary mass and primary energy. For all these reasons, the parametrisation of $G(\theta)$ is expected to depend on both the primary mass and primary energy.

To probe these influences, we repeat the same chain of end-to-end simulations using proton showers at energies of $50 \mathrm{EeV}$ and iron showers at $5 \mathrm{EeV}$. Results in terms of the distortions of the observed event rate $N(\delta)$ are shown in Fig. 11. We also display in the same graph the results obtained using the hadronic interaction model QGSJETII [19]. The differences with respect to the reference model are small, so that the consequences on large scale anisotropy searches presented in Section 5 remain unchanged within the statistics available at the Pierre Auger Observatory.

In addition, there are discrepancies in the hadronic interaction model predictions regarding the number of muons in shower simulations and what is found in our data 20. Higher number of muons influences the weight of the muonic contribution to $S(1000)$. The consequences of increasing the number of muons by a factor of 2 on the distortions of the observed event rate are also shown in Fig. 11. As the muonic contribution to $S(1000)$ is already large at high zenith angles in the reference model, this increase of the number of muons does not lead to large differences.

\section{Conclusion}

In this work, we have identified and quantified a systematic uncertainty affecting the energy determination of cosmic rays detected by the surface detector array of the Pierre Auger Observatory. This systematic uncertainty, induced by the influence of the geomagnetic field on the shower development, has a strength which depends on both the zenith and the azimuthal angles. Consequently, we have shown that it induces distortions of the estimated cosmic ray event rate at a given energy 
at the percent level in both the azimuthal and the declination distributions, the latter of which mimics an almost dipolar pattern.

We have also shown that the induced distortions are already at the level of the statistical uncertainties for a number of events $N \simeq 32000$ (we note that the full Auger surface detector array collects about 6500 events per year with energies above $3 \mathrm{EeV}$ ). Accounting for these effects is thus essential with regard to the correct interpretation of large scale anisotropy measurements taking explicitly profit from the declination distribution.

\section{Acknowledgements}

The successful installation, commissioning, and operation of the Pierre Auger Observatory would not have been possible without the strong commitment and effort from the technical and administrative staff in Malargüe.

We are very grateful to the following agencies and organizations for financial support: Comisión Nacional de Energía Atómica, Fundación Antorchas, Gobierno De La Provincia de Mendoza, Municipalidad de Malargüe, NDM Holdings and Valle Las Leñas, in gratitude for their continuing cooperation over land access, Argentina; the Australian Research Council; Conselho Nacional de Desenvolvimento Científico e Tecnológico (CNPq), Financiadora de Estudos e Projetos (FINEP), Fundação de Amparo à Pesquisa do Estado de Rio de Janeiro (FAPERJ), Fundação de Amparo à Pesquisa do Estado de São Paulo (FAPESP), Ministério de Ciência e Tecnologia (MCT), Brazil; AVCR AV0Z10100502 and AV0Z10100522, GAAV KJB100100904, MSMT-CR LA08016, LC527, 1M06002, and MSM0021620859, Czech Republic; Centre de Calcul IN2P3/CNRS, Centre National de la Recherche Scientifique (CNRS), Conseil Régional Ile-de-France, Département Physique Nucléaire et Corpusculaire (PNC-IN2P3/CNRS), Département Sciences de l'Univers (SDU-INSU/CNRS), France; Bundesministerium für Bildung und Forschung (BMBF), Deutsche Forschungsgemeinschaft (DFG), Finanzministerium Baden-Württemberg, Helmholtz-Gemeinschaft Deutscher Forschungszentren (HGF), Ministerium für Wissenschaft und Forschung, NordrheinWestfalen, Ministerium für Wissenschaft, Forschung und Kunst, Baden-Württemberg, Germany; Istituto Nazionale di Fisica Nucleare (INFN), Ministero dell'Istruzione, dell'Università e della Ricerca (MIUR), Italy; Consejo Nacional de Ciencia y Tecnología (CONACYT), Mexico; Ministerie van Onderwijs, Cultuur en Wetenschap, Nederlandse Organisatie voor Wetenschappelijk Onderzoek (NWO), Stichting voor Fundamenteel Onderzoek der Materie (FOM), Netherlands; Ministry of Science and Higher Education, Grant Nos. N N202 200239 and N N202 207238, Poland; Fundação para a Ciência e a Tecnologia, Portugal; Ministry for Higher Education, Science, and Technology, Slovenian Research Agency, Slovenia; Comunidad de Madrid, Consejería de Educación de la Comunidad de Castilla La Mancha, FEDER funds, Ministerio de Ciencia e Innovación and Consolider-Ingenio 2010 (CPAN), Xunta de Galicia, Spain; Science and Technology Facilities Council, United Kingdom; Department of Energy, Contract Nos. DE-AC02-07CH11359, DE-FR02-04ER41300, National Science Foundation, Grant No. 0450696, The Grainger Foundation USA; ALFA-EC / HELEN, European Union 6th Framework Program, Grant No. MEIF-CT2005-025057, European Union 7th Framework Program, Grant No. PIEF-GA-2008-220240, and UNESCO.

\section{Appendix}

The p.d.f. of the first harmonic amplitude for a data set of $N$ points drawn at random over a circle is known to be the Rayleigh distribution. In this appendix, we generalise this distribution to the case of $N$ points being drawn at random on the sphere over the exposure $\omega(\delta)$ of the Pierre Auger Observatory. Assuming the underlying arrival direction distribution to be of the 
form $\Phi(\alpha, \delta)=\Phi_{0}(1+\mathbf{D} \cdot \mathbf{u})$, the components of the dipolar vector $\mathbf{D}$ are related to the multipolar coefficients through

$$
D_{x}=\sqrt{3} \frac{a_{11}}{a_{00}}, \quad D_{y}=\sqrt{3} \frac{a_{1-1}}{a_{00}}, \quad D_{z}=\sqrt{3} \frac{a_{10}}{a_{00}} .
$$

Denoting by $x, y, z$ the estimates of $D_{x}, D_{y}, D_{z}$, the joint p.d.f. $p_{X, Y, Z}(x, y, z)$ can be factorised in the limit of large number of events in terms of three centered Gaussian distributions $N(0, \sigma)$,

$$
p_{X, Y, Z}(x, y, z)=p_{X}(x) p_{Y}(y) p_{Z}(z)=N\left(0, \sigma_{x}\right) N\left(0, \sigma_{y}\right) N\left(0, \sigma_{z}\right),
$$

where the standard deviation parameters can be calculated from the exposure function [18. With the particular exposure function used here, it turns out that numerical integrations lead to $\sigma \simeq$ $1.02 \sqrt{3 / N}$ and $\sigma_{z} \simeq 1.59 \sqrt{3 / N}$. The joint p.d.f. $p_{R, \Delta, A}(r, \delta, \alpha)$ expressing the dipole components in spherical coordinates is obtained from Eq. (19) by performing the Jacobian transformation

$$
\begin{aligned}
p_{R, \Delta, A}(r, \delta, \alpha) & =\left|\frac{\partial(x, y, z)}{\partial(r, \delta, \alpha)}\right| p_{X, Y, Z}(x(r, \delta, \alpha), y(r, \delta, \alpha), z(r, \delta, \alpha)) \\
& =\frac{r^{2} \cos \delta}{(2 \pi)^{3 / 2} \sigma^{2} \sigma_{z}} \exp \left[-\frac{r^{2} \cos ^{2} \delta}{2 \sigma^{2}}-\frac{r^{2} \sin ^{2} \delta}{2 \sigma_{z}^{2}}\right] .
\end{aligned}
$$

From this joint p.d.f., the p.d.f. of the dipole amplitude (declination) is finally obtained by marginalising over the other variables, yielding

$$
\begin{aligned}
& p_{R}(r)=\frac{r}{\sigma \sqrt{\sigma_{z}^{2}-\sigma^{2}}} \operatorname{erfi}\left(\frac{\sqrt{\sigma_{z}^{2}-\sigma^{2}}}{\sigma \sigma_{z}} \frac{r}{\sqrt{2}}\right) \exp \left(-\frac{r^{2}}{2 \sigma^{2}}\right) \\
& p_{\Delta}(\delta)=\frac{\sigma \sigma_{z}^{2}}{2} \frac{\cos \delta}{\left(\sigma_{z}^{2} \cos ^{2} \delta+\sigma^{2} \sin ^{2} \delta\right)^{3 / 2}} .
\end{aligned}
$$

Finally, one can derive from $p_{R}$ quantities of interest, such as the expected mean noise $\langle r\rangle$, the RMS $\sigma_{r}$ and the probability of obtaining an amplitude greater than $r$ :

$$
\begin{aligned}
\langle r\rangle & =\sqrt{\frac{2}{\pi}}\left(\sigma_{z}+\frac{\sigma^{2} \operatorname{arctanh}\left(\sqrt{1-\sigma^{2} / \sigma_{z}^{2}}\right)}{\sqrt{\sigma_{z}^{2}-\sigma^{2}}}\right) \\
\sigma_{r} & =\sqrt{2 \sigma^{2}+\sigma_{z}^{2}-\langle r\rangle^{2}}, \\
\operatorname{Prob}(>r) & =\operatorname{erfc}\left(\frac{r}{\sqrt{2} \sigma_{z}}\right)+\frac{\sigma}{\sqrt{\sigma_{z}^{2}-\sigma^{2}}} \operatorname{erfi}\left(\frac{\sqrt{\sigma_{z}^{2}-\sigma^{2}}}{\sqrt{2} \sigma \sigma_{z}} r\right) \exp \left(-\frac{r^{2}}{2 \sigma^{2}}\right),
\end{aligned}
$$

which are the equivalent to the well known Rayleigh formulas $\langle r\rangle=\sqrt{\pi / N}, \sigma_{r}=\sqrt{(4-\pi) / N}$ and $\operatorname{Prob}(>r)=\exp \left(-N r^{2} / 4\right)$ when dealing with $N$ points drawn at random over a circle [21.

\section{Acknowledgments}

\section{References}

[1] D. M. Edge et al., J. Phys. A 6 (1973) 1612.

[2] M. Ave, R. A. Vazquez, and E. Zas, Astropart. Phys. 14 (2000) 91.

[3] M. Ave et al., Astropart. Phys. 14 (2000) 109. 
[4] H. Dembinski et al., Astropart. Phys. 34 (2010) 128.

[5] K. Greisen, Ann. Rev. Nuc. Sci. 10 (1960) 63.

[6] K. Kamata and J. Nishimura, Prog. Theor. Phys. 6 (1958) 93.

[7] A. Ivanov et al., JETP Letters 69 (1999) 288.

[8] P. Bernardini et al. for the ARGO-YBJ Collaboration, Proceedings of the $32^{\text {nd }}$ ICRC, Beijing, China, arXiv:1110.0670; H.H. He et al., Proceedings of the $29^{\text {th }}$ ICRC, Pune, India

[9] J. Abraham et al. [Pierre Auger Collaboration], Nucl. Instrum. Meth. A 523 (2004) 50.

[10] J. Abraham et al. [Pierre Auger Collaboration], Nucl. Instr. and Meth. A 613 (2010) 29-39.

[11] J. Abraham et al. [Pierre Auger Collaboration], Phys. Rev. Lett. 101 (2008) 061101.

[12] National Geography Data Center, http://www.ngdc.noaa.gov/seg/geomag/geomag.shtml, 2007.

[13] X. Bertou, et al., Pierre Auger Collaboration, Nucl. Instr. and Meth. A 568 (2006) 839.

[14] S.J. Sciutto, Proceedings of the $27^{\text {th }}$ ICRC, Hamburg, Germany, arXiv:astro-ph/0106044v1.

[15] N.N. Kalmykov and S.S. Ostapchenko, Yad. Fiz. 56 (1993) 105; N.N. Kalmykov, S.S. Ostapchenko, and A.I. Pavlov, Nucl. Phys. B Proc. Suppl. 52B (1997) 17.

[16] D. Heck et al., Report FZKA 6019, Karlsruhe, Germany, 1998.

[17] P. Sommers, Astropart. Phys. 14 (2001) 271.

[18] P. Billoir and O. Deligny, JCAP 02 (2008) 009.

[19] S.S. Ostapchenko, Nucl. Phys. B Proc. Suppl. 151 (2006) 147-150.

[20] R. Engel for the Pierre Auger collaboration, Proceedings of the $30^{t h}$ ICRC, Merida, Mexico, arXiv:0706.1921.

[21] J. Linsley, Phys. Rev. Lett. 34 (1975) 1530. 